\title{
Surface Integrity of Ultrasonically-Assisted Milled Ti6Al4V Alloy Manufactured by Selective Laser Melting
}

Sai Guo ${ }^{1}$, Wei Du², Qinghong Jiang ${ }^{1}$, Zhigang Dong ${ }^{3}$ and Bi Zhang ${ }^{1 *}$

\begin{abstract}
The Ti6AI4V parts produced by the existing selective laser melting (SLM) are mainly confronted with poor surface finish and inevitable interior defects, which substantially deteriorates the mechanical properties and performances of the parts. In this regard, ultrasonically-assisted machining (UAM) technique is commonly introduced to improve the machining quality due to its merits in increasing tool life and reducing cutting force. However, most of the previous studies focus on the performance of UAM with ultrasonic vibrations applied in the tangential and feed directions, whereas few of them on the impact of ultrasonic vibration along the vertical direction. In this study, the effects of feed rate on surface integrity in ultrasonically-assisted vertical milling (UAVM) of the Ti6Al4V alloy manufactured by SLM were systemically investigated compared with the conventional machining (CM) method. The results revealed that the milling forces in UAVM showed a lower amplitude than that in CM due to the intermittent cutting style. The surface roughness values of the parts produced by UAVM were generally greater than that by CM owing to the extra sinusoidal vibration textures induced by the milling cutter. Moreover, the extra vertical ultrasonic vibration in UAVM was beneficial to suppressing machining chatter. As feed rate increased, surface microhardness and thickness of the plastic deformation zone in CM raised due to more intensive plastic deformation, while these two material properties in UAVM were reduced owing to the mitigated impact effect by the high-frequency vibration of the milling cutter. Therefore, the improved surface microhardness and reduced thickness of the subsurface deformation layer in UAVM were ascribed to the vertical high-frequency impact of the milling cutter in UAVM. In general, the results of this study provided an in-depth understanding in UAVM of Ti6AI4V parts manufactured by SLM.
\end{abstract}

Keywords: Ultrasonically-assisted vertical milling, Surface integrity, Ti6Al4V, Selective laser melting

\section{Introduction}

Titanium alloy Ti6Al4V has been widely applied to aviation, aerospace, chemical, and biomedical industries due to its superior mechanical properties and chemical inertness [1]. However, Ti6Al4V, as a typical difficultto-machine material, has rather poor machinability due to its high chemical reactivity, low elasticity modulus, and inferior thermal conductivity, which substantially

\footnotetext{
*Correspondence: zhangb@sustech.edu.cn

${ }^{1}$ Department of Mechanical and Energy Engineering, Southern University

of Science and Technology, Shenzhen 518055, China

Full list of author information is available at the end of the article
}

impedes its practical applications [2]. In order to circumvent these problems, selective laser melting (SLM) has been developed [3]. After an extensive study on SLM, Ngo et al. [4] summarized that SLM possessed many advantages for efficiently building parts with freedom of design, mass customization, waste minimization, fast prototyping, as well as high geometric and material complexities [4-6]. Unfortunately, because SLM is still at the unmatured stage, the parts produced by the current SLM are always confronted with relatively poor surface finish, inevitable interior defects, low dimensional and geometric accuracies, which directly lead to inferior mechanical properties and poor service performances [4]. Among 
these drawbacks of SLM, the surface quality of a critical structural part substantially influences the service performances of the part, especially under conditions of high temperature, complex stress loads, and other extreme environments. In this regard, the parts built by SLM still need to be machined to eliminate the surface unevenness for better mechanical properties and service performances.

Although Ti6Al4V alloy components can be processed using conventional machining $(\mathrm{CM})$ methods, they are always confronted with numerous problems such as large cutting forces, inferior machining quality and accuracy, rough machined surface, and severe tool wear [7]. To overcome the problems pertinent to the CM methods, the ultrasonically-assisted machining (UAM) technique, as one of the non-conventional cutting methods, was developed and has been extensively applied. Normally, UAM is carried out with an additional excitation frequency at an amplitude larger than $2 \mu \mathrm{m}$ and an ultrasonic range over $16 \mathrm{kHz}$ exerted to a workpiece or cutting tool in one or two directions [8-11]. To be specific, ultrasonic vibration can be applied along a linear direction (mostly in the feed or tangential direction) in the one-directional UAM. Moreover, ultrasonic vibration can also be applied simultaneously in both the tangential and feed directions in the two-directional UAM, which thus forms a circular or elliptical trajectory [11]. In this way, extremely rapid movements along the specific directions are applied between the interface of the workpiece and cutting tool, thus greatly facilitating material removal and improving cutting performance.

In order to improve the machinability of difficultto-machine materials, extensive studies have been conducted to study the performance of UAM. In the horizontal vibration-assisted micro-milling of TC4 and $6061 \mathrm{~T} 6$ alloys, $\mathrm{Xu}$ et al. [12] reported that ultrasonic vibration effectively reduced milling force and improved machining accuracy. Sui et al. [13] proposed a novel high-speed ultrasonic vibration cutting method (HUVC) for titanium alloys and revealed that an average cutting force of HUVC was decreased by $20 \%-50 \%$. Through experimental data analysis and finite element simulation, Cakir et al. [14] claimed that the main advantage of the ultrasonic-assisted turning method was the reduction in the average cutting force. Besides, other merits apart from the reduced cutting force and improved machining accuracy in UAM were also reported, such as in UAM of Inconel 718 alloy, better surface finish and improved tool life were verified by Nath et al. [15]; Singh et al. [6] attained high surface quality in the ultrasonically-assisted drilling of different $\mathrm{Ti}$ alloys. In addition, analogous findings in force reduction were uncovered as well by Zhang et al. [16] when studying the effects of rotary ultrasonic elliptical end milling on surface integrity of Ti6Al4V. Moreover, Liu et al. [17] investigated the mechanisms of the rotary ultrasonic elliptical machining on surface integrity of Ti6Al4V and found that surface integrity was improved due to the compressive residual stress, work hardening, and plastic deformation in the machined workpiece surface. In general, after reviewing a variety of research on UAM, it is concluded that the UAM methods demonstrate substantially improved machinability over CM in machining of difficult-to-machine materials, such as increased tool life, reduced cutting force, and improved surface integrity [18]. The significantly improved machinability of UAM over CM mainly results from the reduction of the uncut chip thickness and intermittent contact between the workpiece and cutting tool, which extensively works for both brittle and ductile materials.

Regardless of the vast knowledge on UAM, the research focus of most previous studies lies in the performance of UAM with ultrasonic vibrations applied along the tangential and feed directions, few of them along the vertical direction. Hence, it is critical to investigating the surface integrity of an SLM-built part subjected to ultrasonically-assisted vertical milling (UAVM). Based on the background mentioned above, this study aims to investigate the mechanism of surface and subsurface formation in UAVM of Ti6Al4V alloy produced by SLM. In this study, feed rate was taken as the varied parameter, and the impact of feed rate on surface integrity in UAVM of SLM-produced Ti6Al4V alloy was comprehensively investigated, which included the characterizations and intensive analyses of the milling forces, surface topography and roughness, subsurface alterations, as well as surface microhardness. In addition, the relationship between feed rate and surface integrity was also expounded in this study. In general, this study provided an in-depth understanding of surface integrity in UAVM of Ti6Al4V alloy manufactured by SLM.

\section{Experimental Procedures}

\subsection{Workpiece Preparation}

As shown in Figure 1, Ti6Al4V alloy workpieces with dimensions of $38 \mathrm{~mm} \times 3 \mathrm{~mm} \times 12 \mathrm{~mm}$ were built from the commercial Ti6Al4V alloy powders by the zigzag scanning strategy on an SLM machine (Dimetal-100, Laseradd Tech of China).

The process parameters for SLM are displayed in Table 1. The homogeneous spherical Ti6Al4V alloy powders have an average size of Dv50 $=16.72 \mu \mathrm{m}$. The 
(a)
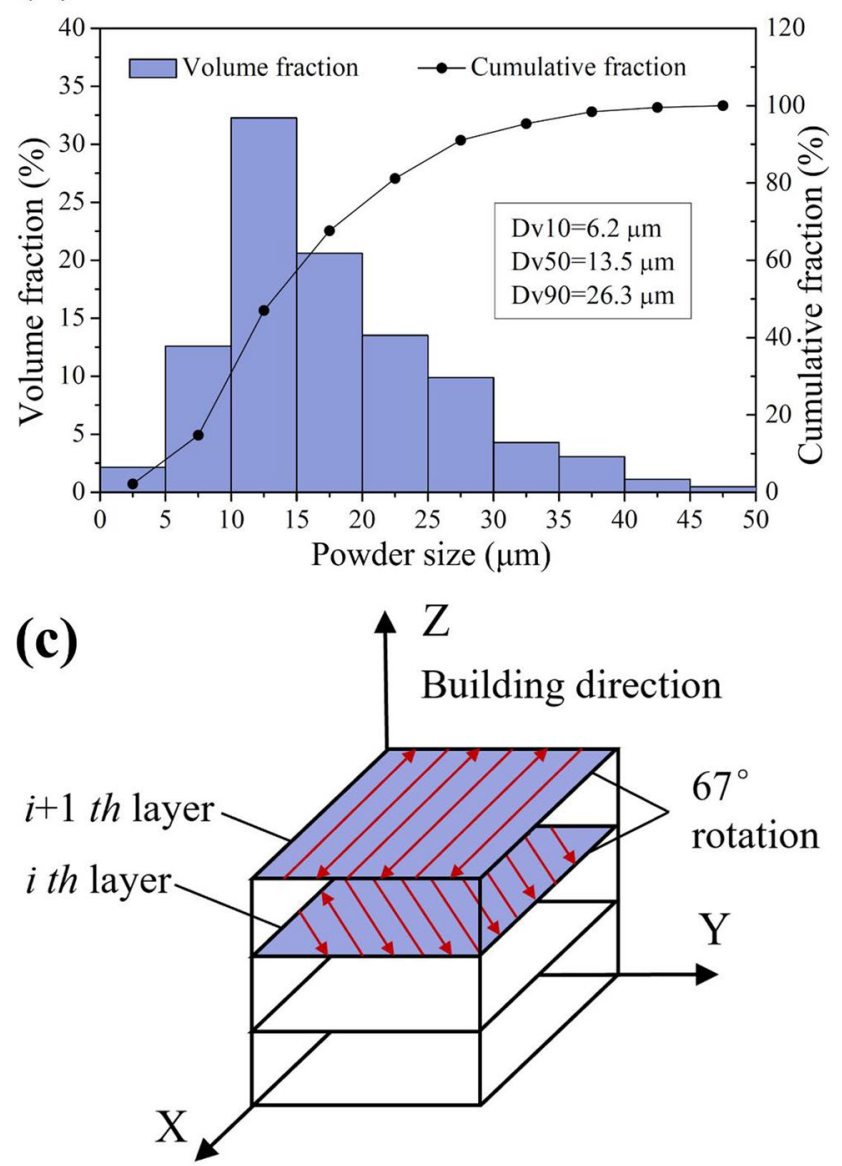

(b)

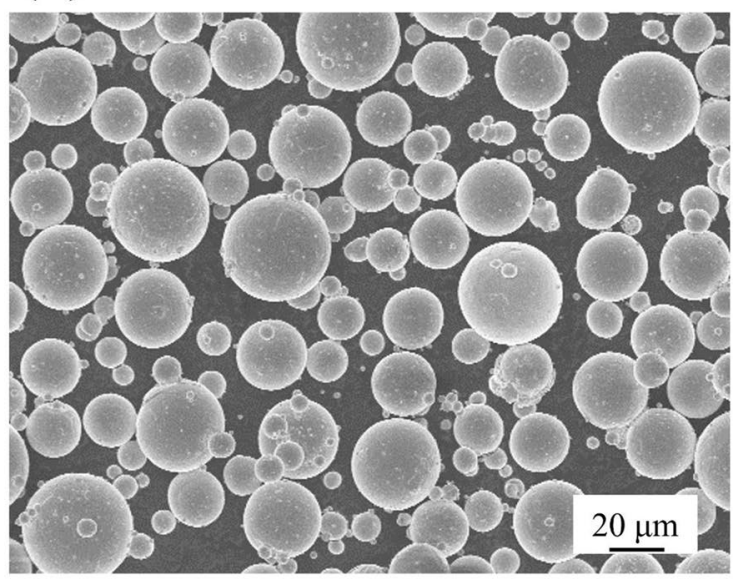

(d)

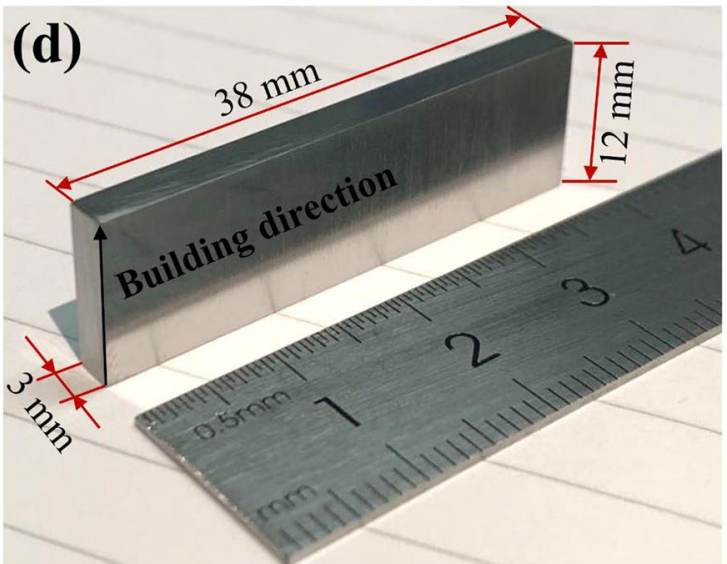

Figure 1 Characteristics of the Ti6AI4V alloy powders and workpiece preparation by SLM: a powder size distribution, b powder morphology, $\mathbf{c}$ scanning strategy of SLM, and $\mathbf{d}$ a workpiece built by SLM

Table 1 Parameters of SLM for building the Ti6Al4V alloy workpieces

\begin{tabular}{lllll}
\hline $\begin{array}{l}\text { Laser power } \\
(\mathbf{W})\end{array}$ & $\begin{array}{l}\text { Layer } \\
\text { thickness } \\
(\mathbf{m m})\end{array}$ & $\begin{array}{l}\text { Scanning } \\
\mathbf{s p e e d} \\
(\mathbf{m m} / \mathbf{s})\end{array}$ & $\begin{array}{l}\text { Hatching } \\
\mathbf{s p a c i n g} \\
(\boldsymbol{\mu m})\end{array}$ & $\begin{array}{l}\text { Scanning } \\
\text { strategy }\end{array}$ \\
\hline 190 & 0.03 & 1000 & 67 & $\begin{array}{l}\text { Zigzag with } \\
67^{\circ} \text { rota- } \\
\text { tion }\end{array}$ \\
\hline
\end{tabular}

Table 2 Element content of the Ti6Al4V alloy powders

\begin{tabular}{llllll}
\hline Element & $\mathbf{A l}$ & $\mathbf{V}$ & $\mathbf{F e}$ & $\mathbf{O}$ & $\mathbf{T i}$ \\
\hline wt.\% & 6.44 & 4.09 & 0.23 & 0.12 & Balance \\
\hline
\end{tabular}

average element content of the Ti6Al4V alloy powders is listed in Table 2. After SLM, a wire-electric discharge machining (wire-EDM) machine was used to remove the manufactured Ti6Al4V alloy workpieces from the substrate. To eliminate the residual stresses induced by the rapid solidification in SLM and obtain good mechanical properties, all workpieces were heated up to $955^{\circ} \mathrm{C}$ and held for $2 \mathrm{~h}$, and then with furnace cooling. After the heat treatment, the workpieces were evaluated to possess Young's modulus of 119 GPa and a maximum elongation of $4.5 \%$ by tensile tests. In addition, all workpieces were ground and polished to the mirror surface for subsequent investigations. The density of the SLM-built Ti6Al4V alloy was measured using a densimeter (Sartorius, Germany), then the relative 

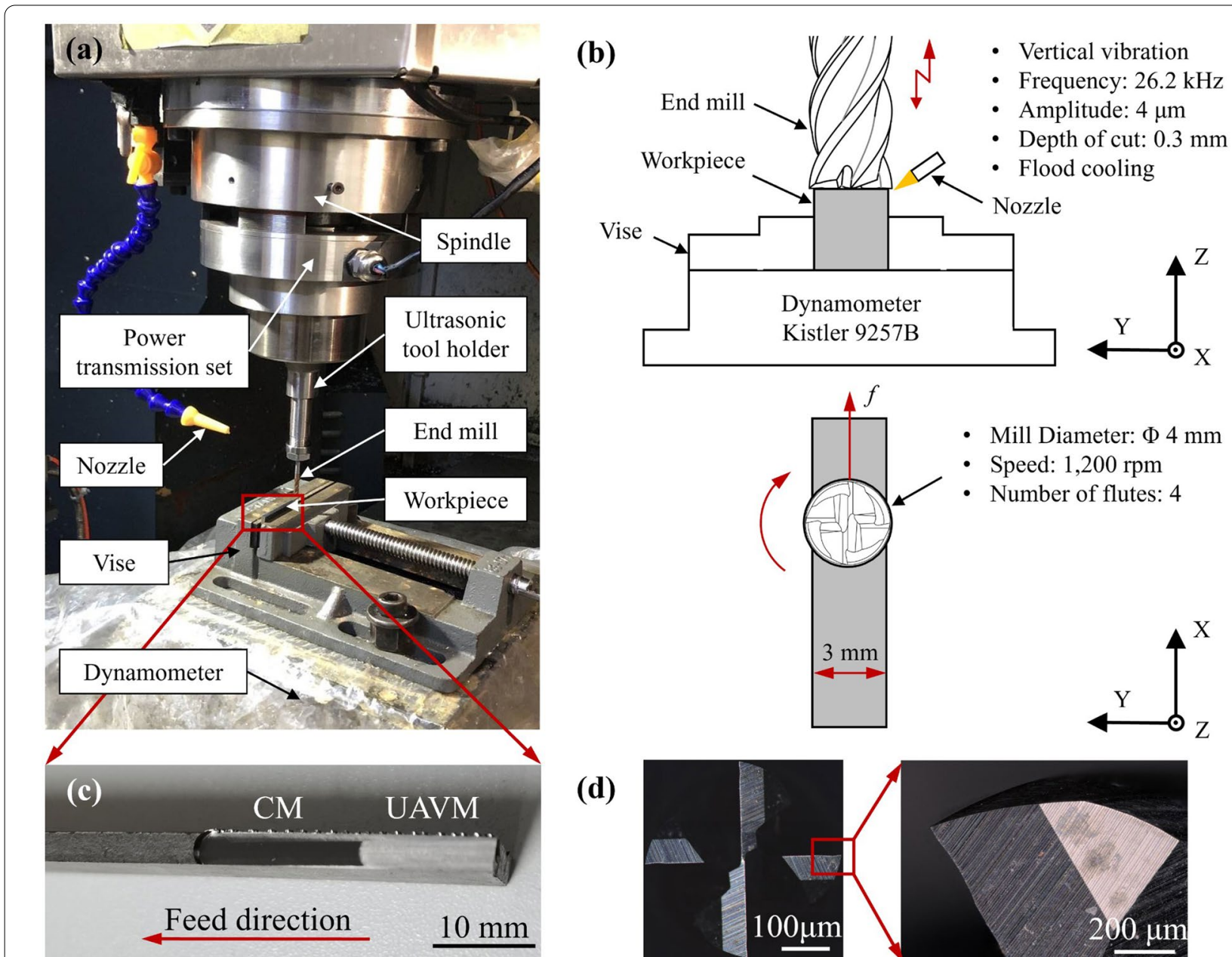

(d)

Figure 2 Experimental setup for the CM and UAVM: a picture of the UAVM setup, $\mathbf{b}$ schematic of the milling process, $\mathbf{c}$ the surfaces machined by the CM and UAVM, $\mathbf{d}$ the cutting edges of the end mill

density was calculated to be $99.1 \%$ by comparing it with the mass density of the as-forged Ti6Al4V alloy.

\subsection{Machining Setup and Procedures}

In this study, a self-built 3-axis CNC machining center equipped with UAVM capabilities was adopted for the CM and UAVM. As illustrated in Figure 2(a), the machining system built by Dong et al. [19] mainly consists of a spindle, an ultrasonic tool holder, and an end mill. In this machining system, the ultrasonic tool holder mounted on the front of the spindle is driven by an ultrasonic actuator inside the tool holder, while the ultrasonic vibration mode is controlled by an external ultrasonic generator which is connected to the ultrasonic vibration milling set by a power transmission set. In this way, the milling experiments both with and without vertical ultrasonic assistance can be realized by simply switching on or off the ultrasonic generator.
A schematic of the milling experiments is shown in Figure 2(b). To facilitate UAVM, an end mill with a larger diameter than the thickness of the workpiece should be employed to reduce the friction from the side edges. In this regard, cemented carbide shoulder end mills

Table 3 Parameters of the end mill

\begin{tabular}{ll}
\hline Manufacturer & Misumi, Japan \\
\hline Type & XCP-CR-EM4R6-R0.3 \\
Material & Cemented carbide \\
Coating & TiSiN \\
Corner radius $(\mathrm{mm})$ & 0.3 \\
Number of cutting edges & 4 \\
Blade diameter $(\mathrm{mm})$ & 4 \\
Helix angle $\left(^{\circ}\right)$ & 40 \\
\hline
\end{tabular}


(Misumi, Japan) with TiSiN coating as demonstrated in Figure 2(d) were employed for both CM and UAVM of the SLM-built Ti6Al4V alloy. The parameters of the end mill are illustrated in Table 3. Each cemented carbide end mill has 4 flutes, a corner radius of $0.3 \mathrm{~mm}$, a blade diameter of $4 \mathrm{~mm}$, and a helix angle of $40^{\circ}$. The typical surfaces machined by the CM and UAVM using the end mill are displayed in Figure 2(c). As the performance of the end milling tests required external equipment, a piezoelectric dynamometer (Kistler 9257B) was employed to collect the signal data of milling forces. The sampling frequency of the force signal was set as $20 \mathrm{kHz}$ in the process due to the limited capacity of the dynamometer.

In calibrating the ultrasonic tool holder, the UAVM and $\mathrm{CM}$ were tuned in advance according to the parameters listed in Table 4, where only the feed rate was changed to investigate its influence on the corresponding machining behaviors. As limited by the configuration of the $\mathrm{CNC}$ machining center, the ultrasonic vibration with a frequency of $26.2 \mathrm{kHz}$ and an amplitude of $4.0 \mu \mathrm{m}$ were only implemented in the tool axial direction which was perpendicular to the machined surface as well as the feed direction, as shown in Figure 2(b). As the width of the workpiece was $3 \mathrm{~mm}$ whilst the end mill diameter was $4 \mathrm{~mm}$, it meant that the radial depth of cut was $100 \%$. Before conducting the UAVM and CM, several milling passes were necessary on each workpiece face to plane the surface, then the UAVM and CM were performed with a distance of $10 \mathrm{~mm}$ successively in one milling pass to ensure the uniformity of milling conditions, as shown in Figure 2(c). In the meanwhile, an oil-based cutting solution was employed in the milling area with a pressure of 6 bars to reduce friction and thus moderate temperature rise.

In addition, for achieving a high reliability of experimental results, triple replicated experiments for each set of parameters were conducted to obtain the average values. For surface and subsurface microstructure observations, representative figures were adopted to compare the differences under different machining parameters.

\subsection{Characterization Methods for the Machined Workpieces}

After end milling, a series of material characterization methods were adopted to study the variation of milling forces and surface integrity of the milled workpieces. The surface topography was captured by using a confocal laser scanning microscope (CLSM, VK-X1000, Keyence, Japan), in which the surface roughness values were directly measured. To determine the microstructure, the finished workpieces were immersed in an etchant with a volume ratio of $\mathrm{HF}: \mathrm{HNO}_{3}: \mathrm{H}_{2} \mathrm{O}=2: 5: 100$ for $10 \mathrm{~s}$, then their microstructures were investigated using a combination of a scanning electron microscope (SEM, Tescan Mira 3, Czech Republic) and the CLSM.

The phase constitution of the workpieces was identified using an X-ray diffractometer (XRD, Smartlab, Rigaku, Japan) at a step size of $0.05^{\circ}$ and a scanning speed of $10^{\circ} \%$ min. As shown in Figure 3, the phase constitution of the workpiece material (SLM-built Ti6Al4V alloy) was determined, where the typical biphasic microstructure was identified with acicular $\alpha$ phase precipitated from the matrix $\beta$ phase. Surface microhardness (HV) tests were performed under a load of $0.3 \mathrm{kgf}$ for a dwell time of $10 \mathrm{~s}$ by using a microhardness tester. In addition, for achieving high reliability of measurement results, triple replicated measurements were taken to obtain the average values. Therefore, the final results were presented as arithmetic means with standard deviations.

\section{Experimental Results}

\subsection{Analysis of Dynamic Milling Forces}

In this study, the machining parameters and methods were performed according to Table 4 . Figure $4 \mathrm{dem}$ onstrates the milling forces at a spindle rotation speed of $n=1200 \mathrm{r} / \mathrm{min}$ within the time-domain in two processing methods. Figure 4 presents that all milling forces in three directions take on an ascending trend with the feed rate although some errors exist in the measurement, whilst $F_{z}$ has the lowest value compared with $F_{x}$ and $F_{y}$. In addition, it is noteworthy that in all the cases, the milling forces in the CM generally demonstrate a larger amplitude than in the UAVM.

Table 4 Parameters for the UAVM and CM

\begin{tabular}{|c|c|c|c|c|c|c|}
\hline No. & Spindle rotation speed $(r / \mathrm{min})$ & $\begin{array}{l}\text { Feed rate } \\
(\mathrm{mm} / \\
\mathrm{min})\end{array}$ & Depth of cut (mm) & Milling distance $(\mathrm{mm})$ & Amplitude of UAVM $(\mu \mathrm{m})$ & Lubrication method \\
\hline 1 & 1200 & 36 & 0.3 & 10 & 4 (UAVM)/0 (CM) & Flood cooling \\
\hline 2 & & 48 & & & & \\
\hline 3 & & 60 & & & & \\
\hline 4 & & 72 & & & & \\
\hline
\end{tabular}



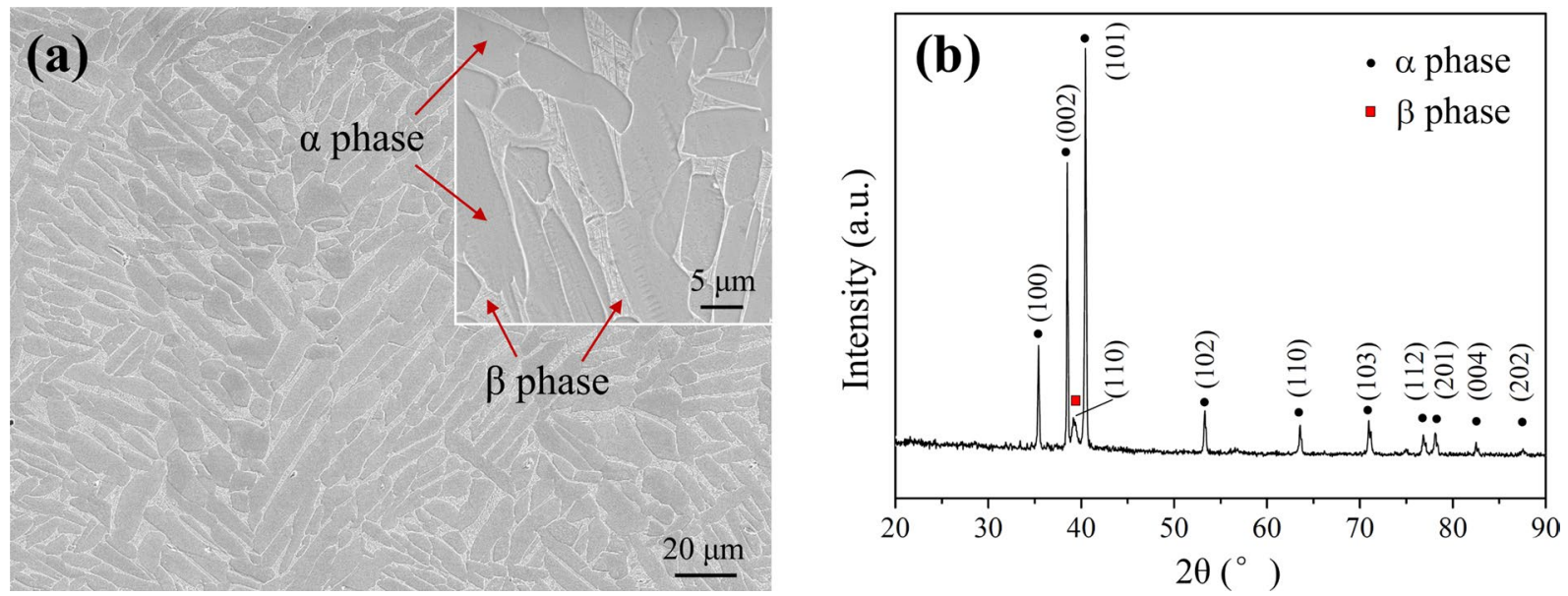

Figure 3 SLM-built Ti6AI4V alloy: a microstructure and $\mathbf{b}$ phase constitution of the SLM-built Ti6AI4V alloy after heat treatment at $955^{\circ} \mathrm{C}$
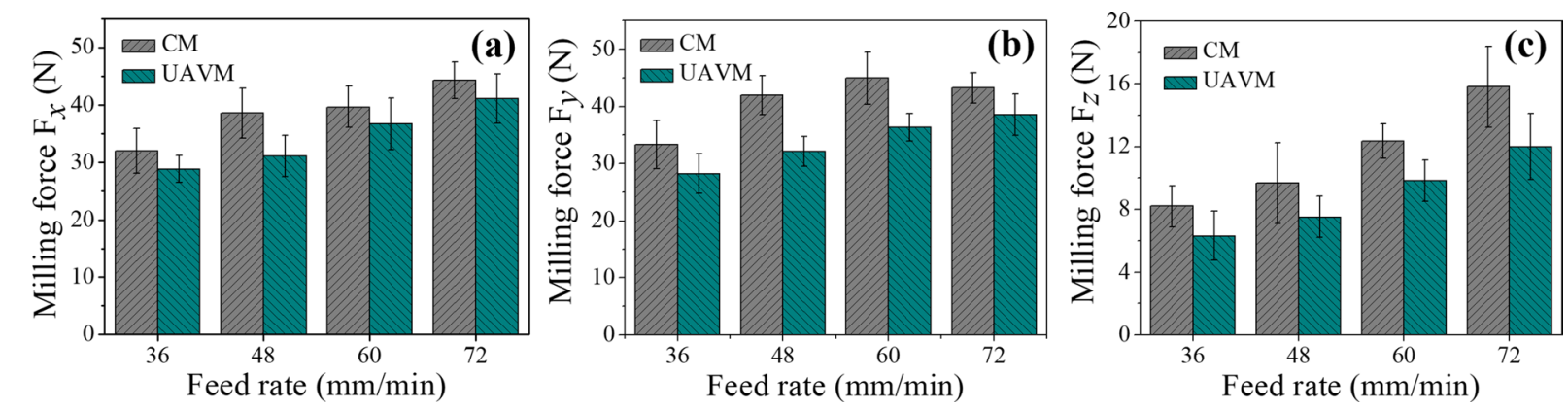

Figure 4 Milling forces against different feed rates $(n=1200 \mathrm{r} / \mathrm{min})$ : $\mathbf{a} F_{x \prime} \mathbf{b} F_{y}$ and $\mathbf{c} F_{z}$

In the process of UAVM, the sampling frequency $(20 \mathrm{kHz})$ of the dynamometer was lower than the vibration frequency $(26.2 \mathrm{kHz})$, which indicated that a sampling period of the dynamometer did not cover a vibration period of the end mill. Therefore, subtle variations in the milling force in UAVM could not be clearly revealed using this dynamometer. In this regard, in order to monitor and study the vibration characteristics of the end mill in the UAVM, a high-resolution laser displacement sensor was adopted accordingly, which had a sampling frequency of $392 \mathrm{kHz}$ (sampling period $2.55 \mu \mathrm{s}$ ) far greater than the designed ultrasonic frequency of $26.2 \mathrm{kHz}$. The ultrasonic vibration signal of the end mill in time-domain was recorded as shown in Figure 5(a), the corresponding spectrum of the ultrasonic vibration signals was also analyzed by the fast Fourier transform (FFT) method, as illustrated in Figure 5(b). It is revealed that the vibration signals fluctuate in a range between $-3.9 \mu \mathrm{m}$ to $3.6 \mu \mathrm{m}$, while the actual resonant frequency of the workpiece in the UAVM is determined to be $26.1 \mathrm{kHz}$. In addition, since
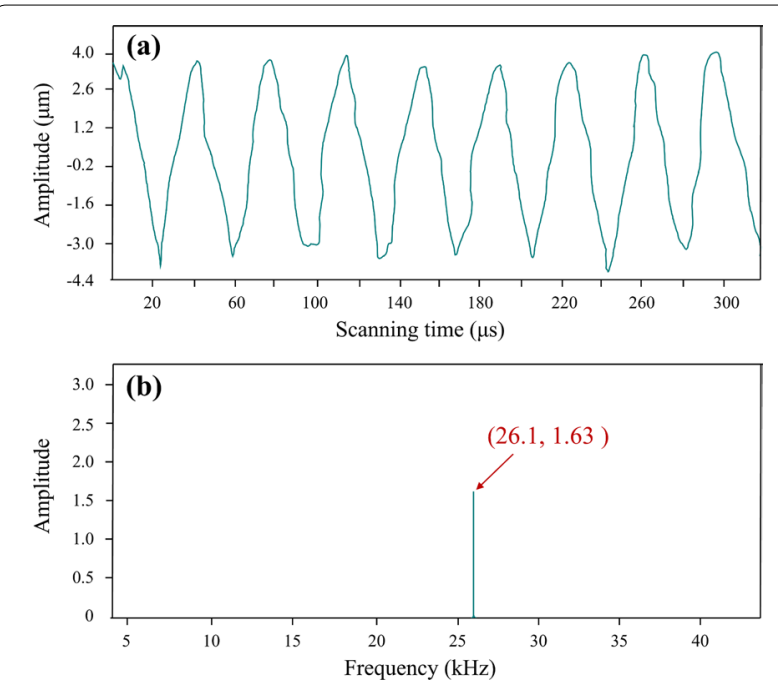

Figure 5 Ultrasonic vibration signal of the end milling process: a ultrasonic vibration signal of the end mill in time-domain, $\mathbf{b}$ the corresponding spectrum of ultrasonic vibration signal processed by FFT 


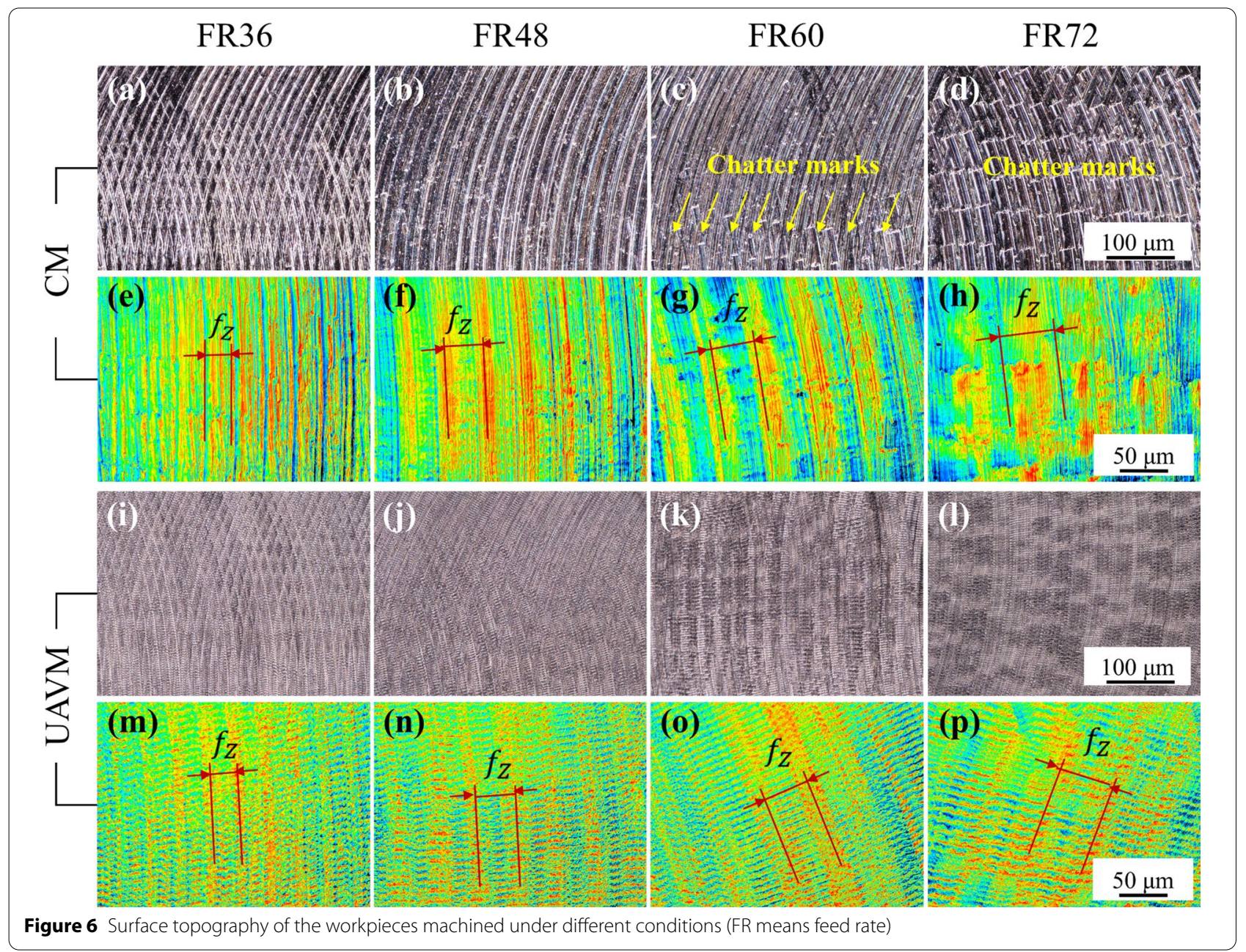

the fluctuation of the vibration signal peaks reflects the overall machining conditions, such as the state of machine vibration, cutting tool runout, and workpiece clamping condition, it is generally used to characterize the vibration instability in machining. The experiment revealed that the frequency spectra demonstrated a low level of vibration noises, which reflected relatively high stability in the CM and UAVM.

\subsection{Surface Topography and Roughness}

A comparison between CM and UAVM on the typically machined surface topography is shown in Figure 6, in which the applied ultrasonic vibration amplitude is $4 \mu \mathrm{m}$ in UAVM. As shown in Figure 6(e), (f), (g) and (h), substantial tool feed trajectories with ridged textures clearly appear on the machined surfaces of CM with the uniform spacing of ridges along the feed direction. The distance between the two feed trajectories is equal to feed per tooth $\left(f_{Z}\right)$. Besides the ridged textures due to the feeding of the cutting edges, the relatively fine sinusoidal vibration textures appear between the feed trajectories on the machined surfaces of UAVM, as shown in Figure 6(m), (n), (o) and (p). In addition, it is worth noticing that Figure 6(c) shows slight chatter marks along the cutter edge trajectory, while distinct chatter marks appear on the machined surface when the feed rate is equal to $72 \mathrm{~mm} /$ min in CM as shown in Figure 6(d).

To further analyze the characteristics of the machined surfaces, line profiles of the surfaces were extracted using the CLSM. Figure 7(a) and (b) display the line-scanning profiles of surface morphology under different machining conditions, and it is revealed that the line-scanning profiles of UAVM surfaces appear greater fluctuations than those generated by CM. For quantitatively characterizing the surface roughness of the machined workpieces, the corresponding area roughness $(\mathrm{Sa}$ and $\mathrm{Sz})$ and profile roughness $(\mathrm{Ra}$ and $\mathrm{Rz})$ along the feed direction are collectively shown in Figure 7(c), (d), (e) and (f). It can be 
(a)

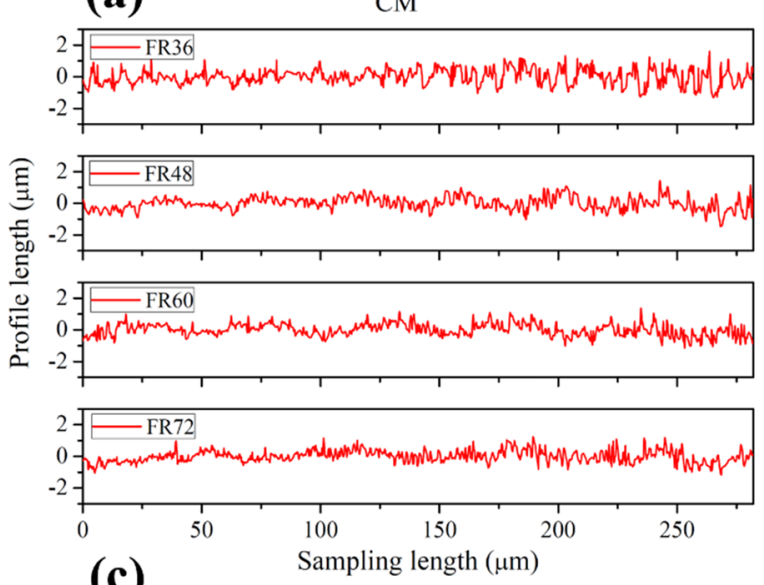

(c)

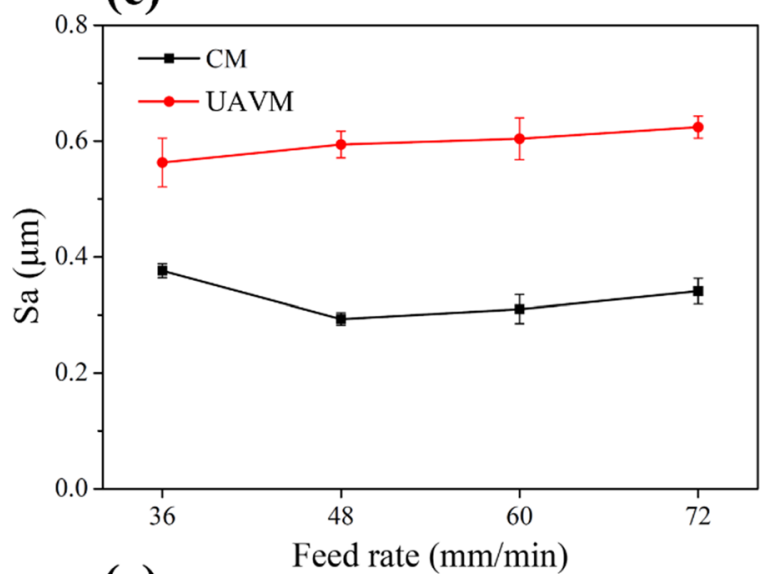

(e)

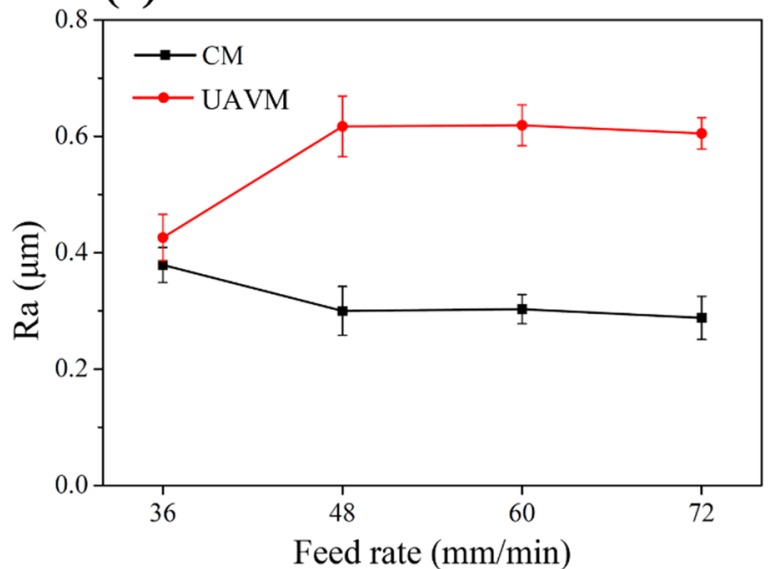

(b) UAVM
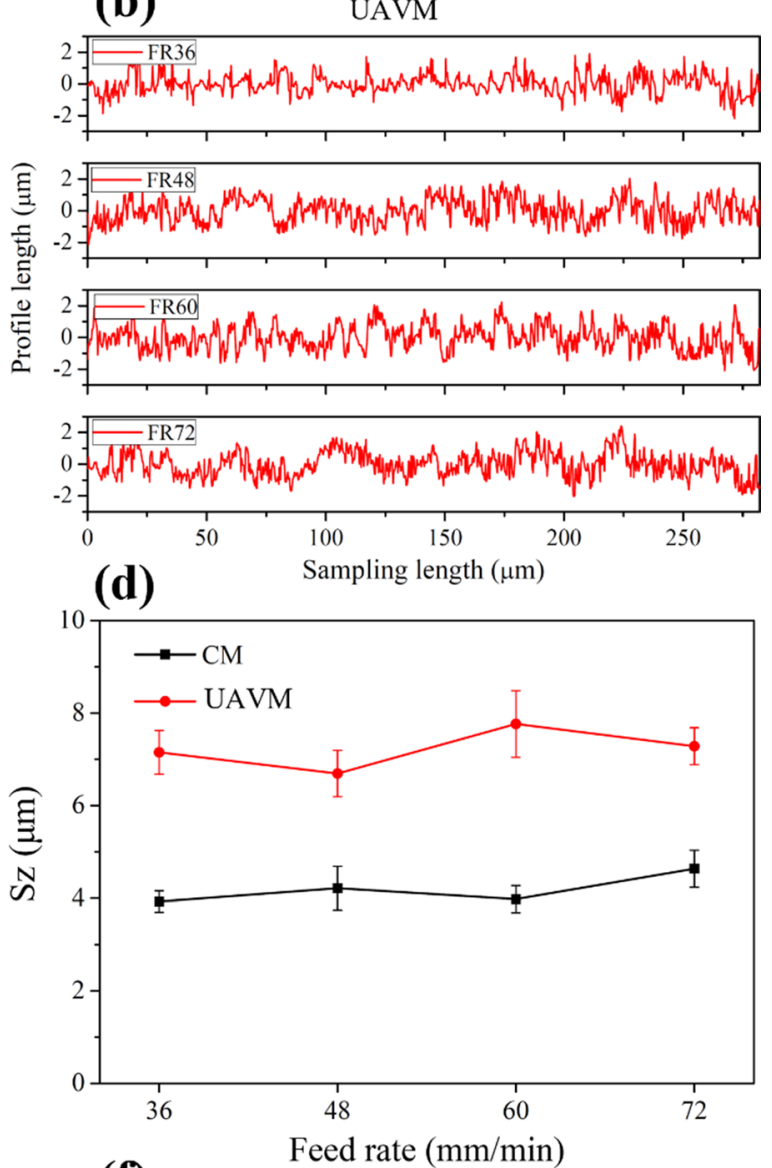

(f)

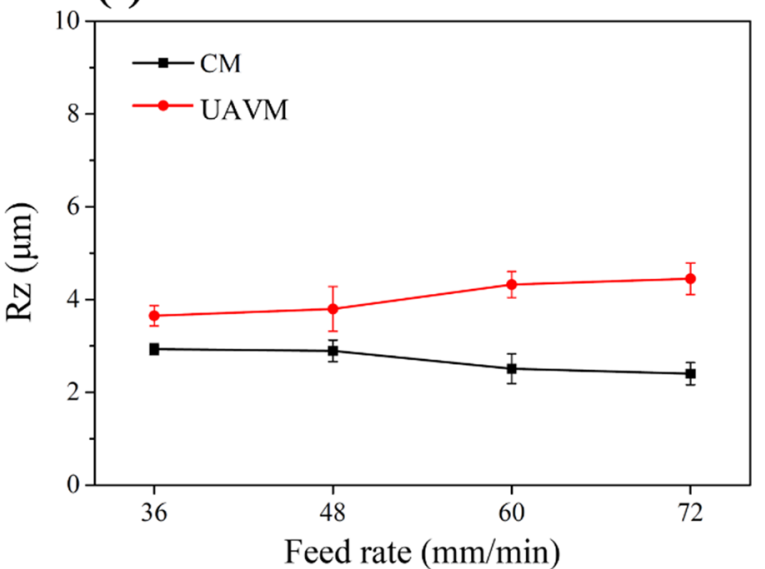

Figure 7 Surface profiles and surface roughness: $\mathbf{a}$ and $\mathbf{b}$ are the line-scanning profiles of surfaces machined under different conditions after CM and UAVM, respectively, $\mathbf{c}$ and $\mathbf{d}$ are the corresponding area roughness (Sa and Sz), e and $\mathbf{f}$ are the corresponding profile roughness (Ra and Rz)(FR means feed rate)

observed that the roughness values of CM and UAVM surfaces remain almost stable with feed rate. However, it can be found that the roughness values of the surfaces produced by UAVM are generally greater than those by
$\mathrm{CM}$, which is consistent with the line-scanning profiles displayed in Figure 7(a) and (b). 


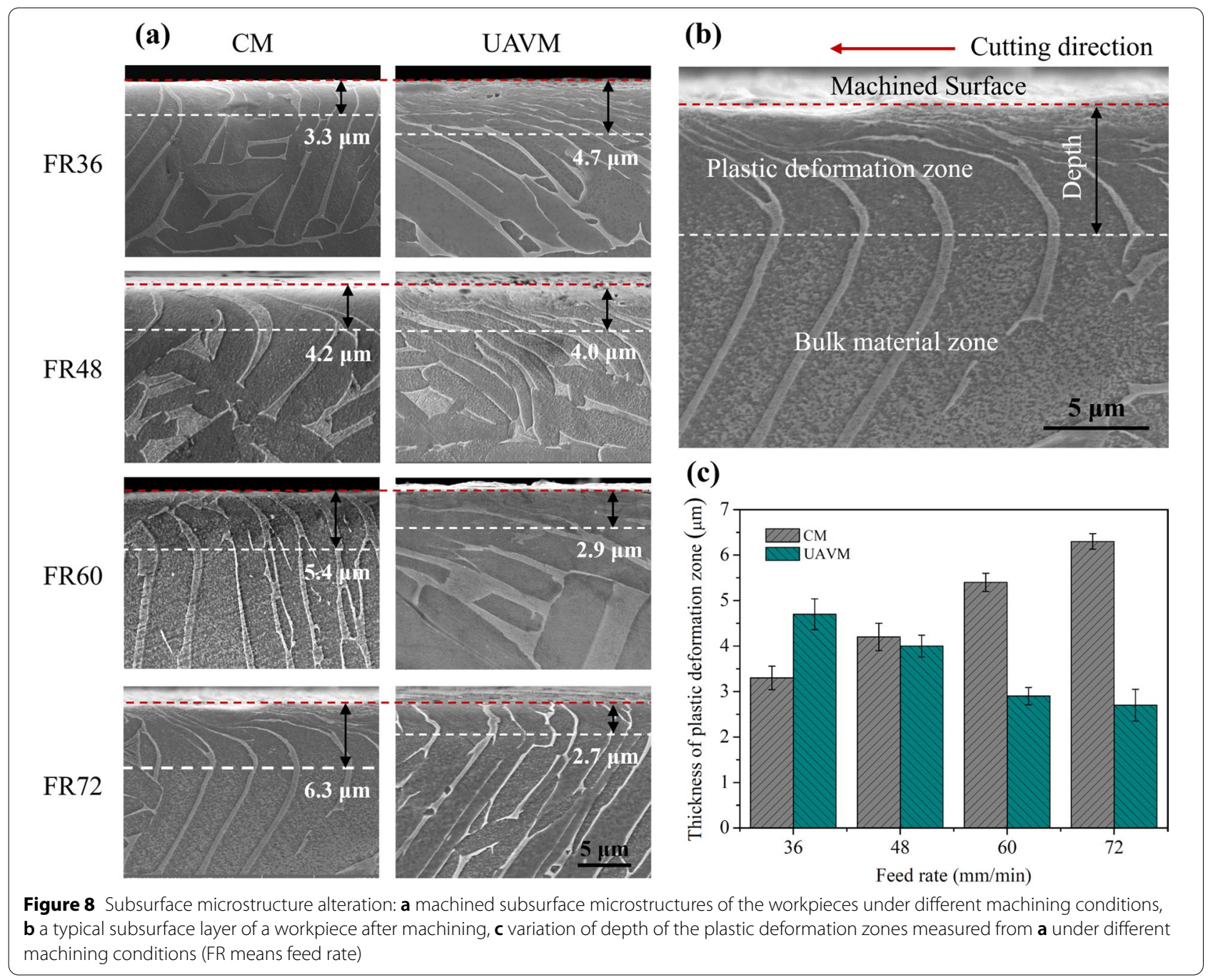

\subsection{Microstructure in Machined Surface}

The cross-sectional views of the subsurface microstructures of the workpieces machined by CM and UAVM under different feed rates are shown in Figure 8(a). From the subsurface microstructures in both CM and UAVM, it can be observed that machining defects, such as cracks, tears, and laps, are absent from all the workpieces tested under the milling conditions. Besides, it can be noticed in Figure 8(b) that all the workpieces machined with different parameters feature with an obvious plastic deformation zone above the undeformed bulk material zone.

The corresponding thickness of the plastic deformation layer is determined in the SEM, and the results are shown in Figure 8(c). In CM, it is revealed that the thickness of the deformation zone is almost doubled from $3.3 \mu \mathrm{m}$ to $6.3 \mu \mathrm{m}$ as the feed rate increases from $36 \mathrm{~mm} / \mathrm{min}$ to $72 \mathrm{~mm} / \mathrm{min}$. In contrast, the thickness variation of the deformation zone in UAVM presents an absolutely different tendency with an increase in feed rate. As shown in

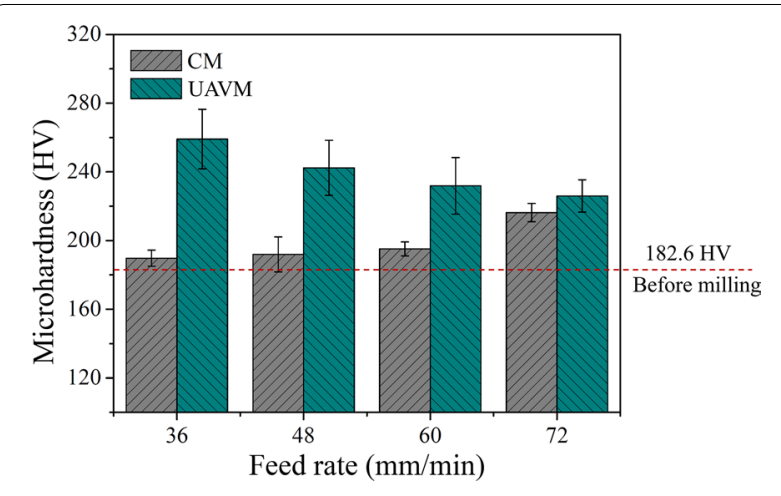

Figure 9 Microhardness of the workpiece surfaces machined under various conditions

Figure 8(a) and (c), the thickness of the deformation zone in UAVM takes on a descending trend, reducing from $4.7 \mu \mathrm{m}$ to $2.7 \mu \mathrm{m}$ as feed rate increases from $36 \mathrm{~mm} / \mathrm{min}$ 
to $72 \mathrm{~mm} / \mathrm{min}$, whereas the plastic deformation zone in the surface machined by UAVM is thicker than that in $\mathrm{CM}$ when feed rate is equal to $36 \mathrm{~mm} / \mathrm{min}$.

\subsection{Surface Microhardness}

Microhardness variations in the workpiece surfaces subjected to different machining conditions are shown in Figure 9. The microhardness of SLM-built Ti6Al4V alloy material before machining is tested to be $182.6 \mathrm{HV}$. As the feed rate increases, the microhardness values of CM surfaces take on an ascending trend, which gradually increases from 189.7 HV to $216.3 \mathrm{HV}$ as feed rate increases from $36 \mathrm{~mm} / \mathrm{min}$ to $72 \mathrm{~mm} / \mathrm{min}$. By contrast, at an increased the feed rate the microhardness of the UAVM surfaces demonstrates a decreasing trend, which gradually decreases from $259.1 \mathrm{HV}$ to $226.0 \mathrm{HV}$ when the feed rate increases from $36 \mathrm{~mm} / \mathrm{min}$ to $72 \mathrm{~mm} / \mathrm{min}$. In addition, it should be noticed that the surfaces produced by UAVM generally show greater microhardness values compared to those produced by CM.

\section{Discussion}

\subsection{Effect of Feed Rate on Milling Forces}

In UAVM, the high-frequency vibration of the milling cutter in the vertical direction can be taken as the forced simple harmonic vibration in the $Z$-axis, while the constant rotating movement of the cutting edge can be regarded as uniform linear motion along the $Y$-axis within a relatively short time and distance. As a result, in UAVM, the relative motions of the cutting edge to the workpiece in the $Z$ - and $Y$-axes are respectively expressed as follows:

$$
\begin{aligned}
& z(t)=a \sin (2 \pi f t), \\
& y(t)=v t,
\end{aligned}
$$

where $a$ and $f$ are the vibration amplitude and vibration frequency of the cutting edge in the $Z$-axis, respectively. $v$ is the instant linear speed of the cutting edge in the $Y$-axis. The relative motion speed of the cutting edge to the workpiece is expressed as follows:

$$
\begin{aligned}
& v_{z}=2 \pi f a \cos (2 \pi f t), \\
& v_{y}=v,
\end{aligned}
$$

In such a manner, the actual motion of the cutting edge in the $Y-Z$ plane is a combined motion of the simple harmonic vibration and uniform linear motion in two perpendicular directions, which demonstrates a sinusoidal cutting locus.

In UAVM, it is commonly believed that lower milling forces over CM are obtained mainly due to the reduced tool-workpiece friction induced by the vibration of the milling cutter [20]. In this study, $F_{x}, F_{y}$, and $F_{z}$ in both UAVM and CM increased with feed rate. It is worth noting that the milling forces for UAVM in three directions, i.e., $F_{x}, F_{y}$, and $F_{z}$, all showed lower amplitudes than those in $\mathrm{CM}$, which could be explained from two perspectives. On one hand, milling forces were substantially influenced by the sinusoidal cutting motion of the cutting edges in UAVM. When the cutting edges vibrated in the vertical direction in UAVM, the end face of the milling cutter intermittently contacted with the workpiece in the highfrequency vibration mode, which could be divided into the cutting stage and the separation stage. Moreover, the dynamic uncut chip thickness in the UAVM method was significantly more complex than the nominal chip thickness in the CM method, which was caused by the complex tool motion trajectories and the separation-stage cutting in UAVM. In consequence, the resultant milling forces were reduced due to the intermittent contact between the end mill and workpiece. On the other hand, the separation space produced between the milling cutter and the workpiece in UAVM could enhance the lubrication with reduced friction, which further facilitated the reduction in milling forces [21]. In addition, for UAVM, the continuous change of relative velocity, relative acceleration and reversed frictional force between a milling cutter and workpiece promoted material removal as well [22].

\subsection{Effect of Feed Rate on Surface Topography and Roughness}

Surface topography substantially affected the bulk properties and service life of material and was hence characterized by surface texture and surface roughness in this study. For CM, it could be found that the tool feed trajectories with uniform spacing of ridges along the feed direction had a spacing distance equal to feed. The width of the adjacent ridges increased with the feed rate, which was considered as the principal factor influencing the machined surface topography in CM. In contrast, because the feed direction was perpendicular to the vibration direction of the end mill in UAVM, the surface texture of the UAVM workpiece was a combined result of the uniform feed trajectories in the feed direction and the sinusoidal texture in the end plane of the cutting tool because of the vertical ultrasonic vibration in UAVM. Moreover, the extension direction of the sinusoidal texture was along the tangential direction of the end mill. As for the distance between the adjacent ridges in the fine sinusoidal vibration textures along the feed direction, it could be determined by the rotational speed and ultrasonic vibration frequency of the milling cutter, which however were set as constant values in this study. Thus, 
the distance between the adjacent ridges along the feed direction was found identical on the machined surfaces. In terms of the fine vibration textures existing between the feed trajectories, their occurrence was attributed to the periodic contact and separation between the end mill and workpiece, which therefore manifested the vital evidence of intermittent machining in UAVM.

In addition, apart from the cutter edge trajectories on the machined surfaces, additional chatter marks appeared along the tangential direction of the end mill, which was caused by the regeneration of waviness of the workpiece surface due to overlapping cuts in CM [23, 24]. As a result of the self-excited vibration, the chatter marks were identified when the feed rate increased from 60 to $72 \mathrm{~mm} / \mathrm{min}$ in $\mathrm{CM}$, which indicated that regenerative chatter was induced due to the increased feed rate. In consequence, the pronounced chatter marks introduced extra unevenness to the machined surfaces as revealed by the increased surface roughness values. In contrast, the surfaces produced by UAVM were covered with the ridged textures and fine sinusoidal vibration textures between feed trajectories rather than chatter marks, which suggested that the vertical ultrasonic vibration was beneficial to suppress the cutter chatter in UAVM.

Surface roughness greatly affects the surface accuracy and quality of a machined workpiece, hence, it was highly essential to consider the surface roughness for assessing the machined surface integrity of the workpiece. In UAVM, an intersection usually formed between two adjacent cutter trajectories due to the cutter feed, while the surface roughness was just determined by the residual height of the intersections. In this study, the steady roughness values indicated that the variation of feed rate from $36 \mathrm{~mm} / \mathrm{min}$ to $72 \mathrm{~mm} / \mathrm{min}$ does not cause significant fluctuation on the height of the trajectories in $\mathrm{CM}$ and UAVM although the corresponding machined surfaces exhibited varied cutting trajectories and textures. However, the deteriorated surface quality with higher surface roughness values was indeed revealed in UAVM compared to CM. Similar results were also reported by Suárez et al. [25] that the surface of Ni-alloy 718 produced by rotary ultrasonic elliptical machining demonstrated Ra roughness value of $25.63 \%$ higher than that machined by CM. In general, the increased Ra roughness values were ascribed to an extra high-frequency vertical vibration introduced to the end mill by UAVM in addition to the horizontal cutting movement in CM, which suggested that another additional material removal mode in the vertical direction was imposed by UAVM. Moreover, Zhang et al. [16] also observed larger surface roughness in rotary ultrasonic elliptical end milling (REUM) of Ti6Al4V compared with CM, which was ascribed to the uniform microtextures mapped on the finished surface of REUM rather than the mechanical surface defect in CM.

In this study, the fine sinusoidal vibration textures between the tool feed trajectories were evidence of the high-frequency material removal mode introduced by UAVM. In this regard, compared with CM, the increased surface roughness values in UAVM were consequences of the extra sinusoidal vibration textures induced by the high-frequency vibration of the end mill.

\subsection{Effect of Feed Rate on Subsurface Microstructure}

The subsurface microstructure of the workpieces subjected to CM and UAVM consisted of two zones: plastic deformation zone and bulk material zone, as similar to that revealed by Zhang et al. in the rotary ultrasonic elliptical end milling of Ti6Al4V alloy [16]. The plastic deformation zone closely located below the machined surface was commonly characterized by rotated grains and deflected grain boundaries along the cutting direction, which generally resulted from intensive plastic deformation induced during the milling process [26]. In contrast, the bulk material zone located beneath the plastic deformation zone remained undisturbed as it was far away from the intensive plastic deformation zone. In this study, the cross-sectional views of the subsurface microstructures machined by CM and UAVM under different feed rates showed no obvious thermally-affected microstructures, such as recasting and white layers, which indicated that the temperature rise in the milling zone should be not higher enough to melt the Ti6Al4V alloy, thus inducing no phase transformations under the various milling conditions.

In terms of the subsurface plastic deformation zone of workpieces machined with CM and UAVM, it displayed the opposite trends with an increasing feed rate. In CM, the increased thickness of the subsurface deformation zone was caused by more intensive plastic deformation induced by the increased feed rate; while the descending thickness of the deformation layer in UAVM indicated that the subsurface plastic deformation was significantly enhanced at a relatively low feed rate, and was however mitigated with an increased feed rate. The thickness variation of the subsurface deformation layer in UAVM can be interpreted in this way: as feed rate increases, the contact time between the end mill and the machining surface in the unit area decreases, which thus undermines the high-frequency impact effect of the ultrasonic vibration on the surface [27]. In this regard, the thickness of the subsurface deformation zone of the UAVM workpieces gradually decreases as a result. In addition, Sun et al. [28] also reported analogous results with an increasing feed rate in end milling of Ti6Al4V alloy. 
It was generally recognized that a mitigated plastic deformation zone in machining signified improved machining quality. In this study, the thickness of the plastic deformation zone on the surfaces machined by $\mathrm{CM}$ and UAVM took on two opposite trends with feed rate, and the surface machined by UAVM displayed a thinner subsurface plastic deformation layer than by $\mathrm{CM}$. Thus, it was concluded that an increased feed rate was beneficial to reducing the thickness of the subsurface plastic deformation layer, thus enhancing the machining quality of SLM alloy by using UAVM.

\subsection{Effect of Feed Rate on Surface Microhardness}

The mechanical properties and service life of a machined part were substantially influenced by the work-hardening effect that resulted from a combination of the uneven stress fields in the machined surfaces. In order to evaluate the effect of surface hardening on the machined surfaces, surface microhardness was usually taken as a crucial indicator. In this study, the surface produced by UAVM generally showed greater microhardness values than by CM due to high-frequency impact of the end mill similar to the findings revealed in the rotary ultrasonic elliptical end milling of Ti6Al4V alloy [16].

As for the effect of cutting heat induced by the end mill, it should be positively correlated with the degree of plastic deformation, which meant that the thicker plastic deformation zone below the milling surface was correlated with server thermal effect. Following this line of reasoning, the workpiece induced with the thicker plastic deformation zone should present lower surface microhardness due to the thermal softening effect, thus representing a negative correlation between them. How, as revealed in Figures 8 and 9, plastic deformation zone thickness and surface microhardness were positively correlated, indicating that thermal impact was insignificant in the process of UAVM and CM in this study. Moreover, as shown in Figure 8, the cross-sectional views of the subsurface microstructures machined by CM and UAVM under different feed rates showed no obvious thermallyaffected microstructures as well, which further verified the unimportant effect of cutting heat in this study.

In terms of the causes of enhanced surface microhardness in UAVM, the high-frequency vibration of the milling cutter introduced a great impact to the cutting zone similar to the effect of shot peening which was a surface modification process commonly adopted to improve the surface hardness and residual compressive stress by shooting micro media onto a workpiece surface at a high speed $[29,30]$. The enhanced surface hardness in shot peening was mainly attributed to the severe plastic deformation in a surface [31]. Similarly, it was concluded that the improved surface microhardness in UAVM was ascribed to the intensive plastic deformation resulting from the high-frequency impact effect of the end mill in UAVM.

Under normal conditions, the microhardness variation and thickness variation of a plastic deformation layer shared a similar trend with an increased feed rate, therefore, the hardened surface should be closely associated with the degree of the subsurface plastic deformation in machining [17]. In this study, it was not difficult to find that feed rate had a marked impact on the surface microhardness of the Ti6Al4V alloy. However, the underlying mechanism between them varied in CM and UAVM. As for $\mathrm{CM}$, the increased surface microhardness value was mainly attributed to the strain hardening resulted from intensive plastic deformation as feed rate increased; while in terms of UAVM, the significant reduction in surface microhardness was directly caused by the decreased contact time between the end mill and the machining surface in a unit area with an increased feed rate. In spite of different mechanisms regarding the origin of surface microhardness in CM and UAVM with an increasing feed rate, the thickness variation of the subsurface plastic deformation layer and the corresponding surface microhardness shared the same trend with an increase in feed rate. Therefore, it was concluded that the thickness of the subsurface plastic deformation layer and the corresponding surface microhardness were positively correlated regardless of the machining methods of CM or UAVM whatsoever.

\section{Conclusions}

In this study, comparative experiments of CM and UAVM were performed on the effects of feed rate on surface integrity of the SLM-built Ti6Al4V alloy. Comprehensive characterization of the machined surfaces and intensive analysis of the milling forces were reported. Based on the experimental results and discussion, the following conclusions are drawn:

1. Milling forces took on an ascending trend with feed rate for both CM and UAVM, while the milling forces in UAVM generally demonstrated a lower amplitude than that in CM due to the decreased friction caused by intermittent contact between the milling cutter and the workpiece.

2. The increased roughness values of the surface produced by UAVM were attained due to the fine sinusoidal vibration textures along the tangential direction of the end mill. In addition, the extra vertical ultrasonic vibration in UAVM was beneficial to suppressing chatter vibrations.

3. The thickness of the deformation zone in CM took on an ascending trend due to more intensive plastic 
deformation induced by the increased feed rate, while the thickness of the deformation zone in UAVM was reduced due to the mitigated high-frequency impact effect of UAVM with the decreased contact time between the end mill and the machining surface in the unit area.

4. The improved surface microhardness and thickness of the subsurface deformation layer in UAVM were ascribed to the vertical high-frequency impact effect of the milling cutter in UAVM. The microhardness variation and the corresponding thickness variation of the plastic deformation layer were positively correlated with an increase in feed rate for both CM and UAVM.

As a result, this study revealed that the introduction of ultrasonic vibration into the conventional milling facilitated material removal and machining quality with reduced milling forces and lower subsurface plastic deformation zone for SLM-built Ti6Al4V, which also promoted the understanding of underlying mechanisms. For practical applications of UAVM of SLM-produced Ti6Al4V, this study suggests that the ultrasonic vibration should be performed with higher amplitude compared with that for brittle materials because the large plasticity of Ti6Al4V will accommodate the highfrequency vibration, thus weakening the beneficial effects of UAVM.

In addition, due to the microstructure and property differences of Ti6Al4V produced by SLM and the conventional methods, the performance of UAVM on Ti6Al4V produced by various methods needs to be compared and explored in future works. Moreover, tool wear of the milling cutter should also be taken into account to comprehensively evaluate the effects of UAVM compared with CM.

\section{Acknowledgements \\ Not applicable.}

\section{Authors' Contributions}

BZ and SG were in charge of the whole trial; SG wrote the manuscript; WD, QJ, and ZD assisted with sampling and laboratory analyses. All authors read and approved the final manuscript.

\section{Authors' Information}

Sai Guo, born in 1993, now is a PhD candidate at Southern University of Science and Technology, China. His research interests focus on high-speed grinding and additive manufacturing.

Wei Du is currently an engineer at Dalian University of Technology, China. He received his PhD degree on mechanical engineering from Dalian University of Technology, China, in 2018. His research interests include direct laser deposition and additive/subtractive hybrid manufacturing.

Qinghong Jiang, born in 1995, a PhD candidate at Southern University of Science and Technology, China. His research interests are selective laser melting and high-speed machining.
Zhigang Dong is currently a professor at Dalian University of Technology, China. His research interests focus on ultraprecision machining and ultrasonicallyassisted vibration machining.

Bi Zhang, is currently a chair professor at Southern University of Science and Technology, China. His research interests include precision machining, and additive/subtractive hybrid manufacturing.

\section{Funding}

Supported by Shenzhen Municipal Science and Technology Innovation Commission of China (Grant Nos. Y01336107, GJHZ20180411143506667, JCYJ20170817111811303).

\section{Competing Interests}

The authors declare no competing financial interests.

\section{Author Details}

${ }^{1}$ Department of Mechanical and Energy Engineering, Southern University of Science and Technology, Shenzhen 518055, China. ${ }^{2}$ School of Mechanical Engineering, Dalian University of Technology, Dalian 116024, China. ${ }^{3}$ Key Laboratory for Precision and Non-Traditional Machining Technology of Ministry of Education, Dalian University of Technology, Dalian 116024, China.

Received: 15 January 2021 Revised: 14 June 2021 Accepted: 18 June 2021 Published online: 05 July 2021

\section{References}

[1] K S Al-Rubaie, S Melotti, A Rabelo, et al. Machinability of SLM-produced Ti6Al4V titanium alloy parts. Journal of Manufacturing Processes, 2020(57): 768-786.

[2] C Ni, L Zhu, Z Yang. Comparative investigation of tool wear mechanism and corresponding machined surface characterization in feed-direction ultrasonic vibration assisted milling of Ti-6Al-4V from dynamic view. Wear, 2019, 436-437: 203006.

[3] M K Thompson, G Moroni, TVaneker, et al. Design for additive manufacturing: Trends, opportunities, considerations, and constraints. CIRP Annals, 2016, 65(2): 737-760.

[4] TD Ngo, A Kashani, G Imbalzano, et al. Additive manufacturing (3D printing): A review of materials, methods, applications and challenges. Composites Part B: Engineering, 2018, 143: 172-196.

[5] S A M Tofail, E P Koumoulos, A Bandyopadhyay, et al. Additive manufacturing: scientific and technological challenges, market uptake and opportunities. Materials Today, 2018, 21(1): 22-37.

[6] R Singh, J S Khamba. Investigation for ultrasonic machining of titanium and its alloys. Journal of Materials Processing Technology, 2007, 183(2): 363-367.

[7] C Ni, L Zhu, C Liu, et al. Analytical modeling of tool-workpiece contact rate and experimental study in ultrasonic vibration-assisted milling of Ti-6Al-4V. International Journal of Mechanical Sciences, 2018, 142-143: 97-111.

[8] X Liu, D Wu, J Zhang, et al. Analysis of surface texturing in radial ultrasonic vibration-assisted turning. Journal of Materials Processing Technology, 2019, 267: 186-195.

[9] E Shamoto, T Moriwaki. Study on elliptical vibration cutting. CIRP Annals, 1994, 43(1): 35-38.

[10] C Zhang, Y Song. Design and kinematic analysis of a novel decoupled $3 \mathrm{D}$ ultrasonic elliptical vibration assisted cutting mechanism. Ultrasonics, 2019, 95: 79-94.

[11] F Ning, W Cong, Ultrasonic vibration-assisted (UV-A) manufacturing processes: State of the art and future perspectives. Journal of Manufacturing Processes, 2020, 51: 174-190.

[12] L Xu, H Na, G Han. Machinablity improvement with ultrasonic vibration-assisted micro-milling. Advances in Mechanical Engineering, 2018, 10(12): 1-12

[13] H Sui, X Zhang, D Zhang, et al. Feasibility study of high-speed ultrasonic vibration cutting titanium alloy. Journal of Materials Processing Technology, 2017, 247: 111-120. 
[14] F H Cakir, S Gurgen, M A Sofuoglu, et al. Finite element modeling of ultrasonic assisted turning of Ti6Al4V alloy. Procedia - Social and Behavioral Sciences, 2015, 195: 2839-2848.

[15] C Nath, M Rahman. Effect of machining parameters in ultrasonic vibration cutting. International Journal of Machine Tools and Manufacture, 2008, 48(9): 965-974.

[16] M Zhang, D Zhang, D Geng, et al. Effects of tool vibration on surface integrity in rotary ultrasonic elliptical end milling of Ti-6Al-4V. Journal of Alloys and Compounds, 2020, 821: 153266.

[17] J Liu, X Jiang, X Han, et al. Effects of rotary ultrasonic elliptical machining for side milling on the surface integrity of Ti-6Al-4V. The International Journal of Advanced Manufacturing Technology, 2018, 101(5-8): 1451-1465.

[18] Z Yang, L Zhu, G Zhang, et al. Review of ultrasonic vibration-assisted machining in advanced materials. International Journal of Machine Tools and Manufacture, 2020, 156: 103594.

[19] Z Dong, F Zheng, X Zhu, et al. Characterization of material removal in ultrasonically assisted grinding of SiCp/AI with high volume fraction. The International Journal of Advanced Manufacturing Technology, 2017, 93(5): 2827-2839.

[20] WXXu, L C Zhang. Ultrasonic vibration-assisted machining: principle, design and application. Advances in Manufacturing, 2015, 3(3): 173-192.

[21] L Zhu, C Ni, Z Yang, et al. Investigations of micro-textured surface generation mechanism and tribological properties in ultrasonic vibrationassisted milling of Ti-6Al-4V. Precision Engineering, 2019, 57: 229-243.

[22] T Moriwaki, E Shamoto. Ultrasonic elliptical vibration cutting. CIRP Annals, 1995, 44(1): 31-34.

[23] G Quintana, J Ciurana. Chatter in machining processes: A review. International Journal of Machine Tools and Manufacture, 2011, 51(5): 363-376.
[24] C Ma, J Ma, E Shamoto, et al. Analysis of regenerative chatter suppression with adding the ultrasonic elliptical vibration on the cutting tool. Precision Engineering, 2011, 35(2): 329-338.

[25] A Suárez, F Veiga, L N L de Lacalle, et al. Effects of ultrasonics-assisted face milling on surface integrity and fatigue life of Ni-Alloy 718. Journal of Materials Engineering and Performance, 2016, 25(11): 5076-5086.

[26] X Li, P Zhao, Y Niu, et al. Influence of finish milling parameters on machined surface integrity and fatigue behavior of Ti1023 workpiece. The International Journal of Advanced Manufacturing Technology, 2017, 91(1): 1297-1307.

[27] M Cheng, D Zhang, H Chen, et al. Development of ultrasonic thread root rolling technology for prolonging the fatigue performance of high strength thread. Journal of Materials Processing Technology, 2014, 214(11): 2395-2401.

[28] J Sun, Y B Guo. A comprehensive experimental study on surface integrity by end milling Ti-6Al-4V. Journal of Materials Processing Technology, 2009, 209(8): 4036-4042.

[29] G Ongtrakulkij, A Khantachawana, K Kondoh. Effects of media parameters on enhance ability of hardness and residual stress of Ti6Al4V by fine shot peening. Surfaces and Interfaces, 2020, 18: 100424.

[30] Q Lin, H Liu, C Zhu, et al. Investigation on the effect of shot peening coverage on the surface integrity. Applied Surface Science, 2019, 489: 66-72.

[31] N Ferreira, J S Jesus, J A M Ferreira, et al. Effect of bead characteristics on the fatigue life of shot peened Al 7475-T7351 specimens. International Journal of Fatigue, 2020, 134: 105521.

\section{Submit your manuscript to a SpringerOpen ${ }^{\circ}$ journal and benefit from:}

- Convenient online submission

- Rigorous peer review

- Open access: articles freely available online

- High visibility within the field

Retaining the copyright to your article

Submit your next manuscript at $\boldsymbol{\nabla}$ springeropen.com 